\title{
Countable products of spaces of finite sets
}

\author{
by
}

\author{
Antonio Avilés (Murcia)
}

\begin{abstract}
We consider the compact spaces $\sigma_{n}(\Gamma)$ of subsets of $\Gamma$ of cardinality at most $n$ and their countable products. We give a complete classification of their Banach spaces of continuous functions and a partial topological classification.
\end{abstract}

For an infinite set $\Gamma$ and a natural number $n$, we consider the space

$$
\sigma_{n}(\Gamma)=\left\{x \in\{0,1\}^{\Gamma}:|\operatorname{supp}(x)| \leq n\right\} .
$$

Here $\operatorname{supp}(x)=\left\{\gamma \in \Gamma: x_{\gamma} \neq 0\right\}$. This is a closed, hence compact subset of $\{0,1\}^{\Gamma}$, which is identified with the family of all subsets of $\Gamma$ of cardinality at most $n$. In this work we will study the spaces which are countable products of spaces $\sigma_{n}(\Gamma)$, mainly their topological classification as well as the classification of their Banach spaces of continuous functions.

Let $T$ be the set of all sequences $\left(\tau_{n}\right)_{n=1}^{\infty}$ with $0 \leq \tau_{n} \leq \omega$. When $\tau$ runs over $T, \sigma_{\tau}(\Gamma)=\prod_{n=1}^{\infty} \sigma_{n}(\Gamma)^{\tau_{n}}$ runs over all finite and countable products of spaces $\sigma_{k}(\Gamma)$. For $\tau \in T$ we will denote by $j(\tau)$ the supremum of all $n$ with $\tau_{n}>0$, and by $i(\tau)$ the supremum of all $n$ with $\tau_{n}=\omega$. If $\tau_{n}<\omega$ for all $n \geq 1$, then $i(\tau)=0$. Always $0 \leq i(\tau) \leq j(\tau) \leq \omega$. Theorem 1 below summarizes our knowledge about the topological classification and its proof consists of a number of lemmas given in Section 2.

THEOREM 1. Let $\tau, \tau^{\prime} \in T$ and $\Gamma$ an uncountable set.

(1) Suppose $j(\tau)<\omega$. In this case, $\sigma_{\tau^{\prime}}(\Gamma)$ is homeomorphic to $\sigma_{\tau}(\Gamma)$ if and only if $i(\tau)=i\left(\tau^{\prime}\right)$ and $\tau_{n}=\tau_{n}^{\prime}$ for all $n>i(\tau)$.

(2) Suppose $i(\tau)=\omega$. In this case, if $i\left(\tau^{\prime}\right)=\omega$, then $\sigma_{\tau}(\Gamma)$ is homeomorphic to $\sigma_{\tau^{\prime}}(\Gamma)$.

2000 Mathematics Subject Classification: 46B50, 46B26, 54B10, 54D30, $46 \mathrm{~B} 03$.

Key words and phrases: uniform Eberlein compact, regular averaging operator, countable product.

This research was partially supported by the grant BFM2002-01719 of MCyT (Spain) and a FPU grant of MEC (Spain). 
This is not a complete classification and leaves the following question open:

Problem 1. Let $\Gamma$ be an uncountable set and $\tau, \tau^{\prime} \in T$ such that $j\left(\tau^{\prime}\right)=$ $j(\tau)=\omega, i(\tau)<\omega$ and there exists some $n \geq i(\tau)$ with $\tau_{n} \neq \tau_{n}^{\prime}$. Is $\sigma_{\tau}(\Gamma)$ homeomorphic to $\sigma_{\tau^{\prime}}(\Gamma)$ ?

For example, one particular instance of the problem is whether $\prod_{i=1}^{\infty} \sigma_{i}(\Gamma)$ is homeomorphic to $\prod_{i=2}^{\infty} \sigma_{i}(\Gamma)$.

About the spaces of continuous functions, it has recently been proved by Marciszewski [6] that a Banach space $C(K)$ ( $K$ a compact space) is isomorphic to $c_{0}(\Gamma)$ if and only if $K \subset \sigma_{n}(\Gamma)$ for some $n<\omega$. This is the case of any compact space of the form $K=\prod_{i=1}^{n} \sigma_{k_{i}}(\Gamma)$ which can be embedded into $\sigma_{\sum k_{i}}\left(\bigcup_{i=1}^{n} \Gamma \times\{i\}\right)$ by $x \mapsto \bigcup_{i=1}^{n} x_{i} \times\{i\}$. Hence, it is a consequence of Marciszewski's result that the Banach spaces of continuous functions over finite products of spaces $\sigma_{k}(\Gamma)$ over a fixed $\Gamma$ are all isomorphic. In Section 1 we prove a similar result for countable products:

THEOREM 2. Let $\Gamma$ be an infinite set and $\left(k_{n}\right)$ be any sequence of positive integers. Then the Banach spaces $C\left(\prod_{n<\omega} \sigma_{k_{n}}(\Gamma)\right)$ and $C\left(\sigma_{1}(\Gamma)^{\omega}\right)$ are isomorphic.

The techniques that we will use are based on the use of regular averaging operators and the so called Pełczyński's decomposition method, developed in [8] and [9] in order to prove Milyutin's result that the spaces of continuous functions over uncountable metrizable compacta are all isomorphic.

Definition 3. Let $\phi: L \rightarrow K$ be a continuous surjection between compact spaces. A regular averaging operator for $\phi$ is a bounded positive linear operator $T: C(L) \rightarrow C(K)$ with $T\left(1_{L}\right)=1_{K}$ and $T(x \circ \phi)=x$ for all $x \in C(K)$.

The countable products of spaces of the form $\sigma_{n}(\Gamma)$ are uniform Eberlein compact spaces (cf. [3]). This class consists of all weakly compact subsets of Hilbert spaces, or equivalently of all compact subsets of the spaces

$$
B(\Gamma)=\left\{x \in[-1,1]^{\Gamma}: \sum_{\gamma \in \Gamma}\left|x_{\gamma}\right| \leq 1\right\} \sim\left(B_{\ell_{2}(\Gamma)}, w\right)
$$

for some set $\Gamma$. Indeed, $\sigma_{n}(\Gamma)$ is homeomorphic to $B(\Gamma) \cap\{0,1 / n\}^{\Gamma}$. We establish the following result:

THEOREM 4. Let $K$ be a uniform Eberlein compact space of weight $\kappa$. Then there is a closed subspace $L$ of $\sigma_{1}(\kappa)^{\mathbb{N}}$ and an onto continuous map $f: L \rightarrow K$ which admits a regular averaging operator.

This improves a result of Argyros and Arvanitakis [1] that for every uniform Eberlein compact space $K$ there is a totally disconnected uniform 
Eberlein compact space $L$ of the same weight and a continuous surjection $f: L \rightarrow K$ which admits a regular averaging operator, and also a result of Benyamini, Rudin and Wage [2] that every uniform Eberlein compact space of weight $\kappa$ is a continuous image of a closed subset of $\sigma_{1}(\kappa)^{\mathbb{N}}$. We note that there are many totally disconnected uniform Eberlein compact spaces which cannot be embedded into $\sigma_{1}(\kappa)^{\mathbb{N}}$ (cf. Lemma 12 below).

Notations. All topological spaces will be assumed to be completely regular. By identifying elements of $\{0,1\}^{\Gamma}$ with subsets of $\Gamma$, the space $\sigma_{n}(\Gamma) \subset\{0,1\}^{\Gamma}$ can be viewed as the family of all subsets of $\Gamma$ of cardinality less than or equal to $n$, endowed with the topology which has a base the sets of the form

$$
\Phi_{F}^{G}=\left\{y \in \sigma_{n}(\Gamma): F \subset y \subset \Gamma \backslash G\right\}
$$

for $F$ and $G$ finite subsets of $\Gamma$. We will denote by $p: \sigma_{1}(\Gamma)^{k} \rightarrow \sigma_{k}(\Gamma)$ the continuous surjection given by

$$
p\left(x_{1}, \ldots, x_{k}\right)=x_{1} \cup \cdots \cup x_{k} .
$$

Note that from the existence of such a function it follows that any countable product $\prod_{i<\omega} \sigma_{k_{i}}(\Gamma)$ is a continuous image of $\sigma_{1}(\Gamma)^{\omega}$. We will also write $B^{+}(\Gamma)=B(\Gamma) \cap[0,1]^{\Gamma}$.

1. Banach space classification. The following Theorem 5 is the key result of this section. A somewhat similar fact can be found in [10], namely that the natural surjection $K^{2} \rightarrow \exp _{2}(K)=\{\{x, y\}: x, y \in K\}$ given by $(x, y) \mapsto\{x, y\}$ has a regular averaging operator.

TheOREM 5. The map $p: \sigma_{1}(\Gamma)^{k} \rightarrow \sigma_{k}(\Gamma)$ admits a regular averaging operator.

Proof. For every $y \in \sigma_{k}(\Gamma)$ denote by $L(y)$ the subset of $p^{-1}(y)$ consisting of all $\left(x^{1}, \ldots, x^{k}\right) \in p^{-1}(y)$ such that $x^{i} \cap x^{j}=\emptyset$ for $i \neq j$ (that is, $L(y)$ consists of those tuples of $p^{-1}(y)$ in which no singleton appears twice).

The regular averaging operator $T: C\left(\sigma_{1}(\Gamma)^{k}\right) \rightarrow C\left(\sigma_{k}(\Gamma)\right)$ is defined as follows:

$$
T(f)(y)=\frac{1}{|L(y)|} \sum_{x \in L(y)} f(x) .
$$

The only difficult point is to prove that $T(f)$ is a continuous function whenever $f$ is continuous. So fix $f \in C\left(\sigma_{1}(\Gamma)^{k}\right)$ and a point $y \in \sigma_{k}(\Gamma)$ and $\varepsilon>0$. For each $x=\left(x_{1}, \ldots, x_{k}\right) \in L(y)$, since $f$ is continuous at $x$, there is a neighborhood $U_{x}$ of $x$ in $\sigma_{1}(\Gamma)^{k}$ for which $\sup _{x^{\prime} \in U_{x}}\left|f(x)-f\left(x^{\prime}\right)\right|<\varepsilon$. The set $U_{x}$ must contain a basic neighborhood of $x$ of the form

$$
\Phi_{x_{1}}^{G_{1}^{x}} \times \cdots \times \Phi_{x_{k}}^{G_{k}^{x}} \subset U_{x}
$$


where $G_{i}^{x}$ is a finite subset of $\Gamma$ disjoint from $x_{i}$. We define a neighborhood of $y$ as

$$
V=\Phi_{y}^{\bigcup_{x \in L(y)}} \bigcup_{i=1}^{k} G_{i}^{x} \backslash y
$$

and we shall see that $\left|T(f)(y)-T(f)\left(y^{\prime}\right)\right|<\varepsilon$ for every $y^{\prime} \in V$. So we fix $y^{\prime} \in V$ (in particular $y \subset y^{\prime}$ ). First, we define an onto map $r: L\left(y^{\prime}\right) \rightarrow L(y)$ in the following way: if $\left(x_{1}, \ldots, x_{k}\right) \in L\left(y^{\prime}\right)$ then $r(x)=\left(r(x)_{1}, \ldots, r(x)_{k}\right)$ where $r(x)_{i}=x_{i} \cap y$. It is straightforward to check that all the fibers of $r$ have the same cardinality, say $n=\left|r^{-1}(x)\right|$, so that $\left|L\left(y^{\prime}\right)\right|=n|L(y)|$. The key fact (used in the final inequality below) is that if $x \in L(y)$ and $x^{\prime} \in r^{-1}(x)$, then $x^{\prime} \in U_{x}$. To see this, take $x=\left(x_{1}, \ldots, x_{k}\right) \in L(y)$ and $x^{\prime}=\left(x_{1}^{\prime}, \ldots, x_{k}^{\prime}\right) \in r^{-1}(x)$. We check that $x_{i}^{\prime} \in \Phi_{x_{i}}^{G_{i}^{x}}$. If $x_{i}^{\prime} \subset y$ then $x_{i}^{\prime}=x_{i}$. If $x_{i}^{\prime}=\{\gamma\} \subset y^{\prime} \backslash y$ then $x_{i}=\emptyset$ and since $y^{\prime} \in V$, we have $\gamma \notin G_{i}^{x}$ and again $x_{i}^{\prime} \in \Phi_{x_{i}}^{G_{i}^{x}}$. Finally,

$$
\begin{aligned}
\left|T(f)\left(y^{\prime}\right)-T(f)(y)\right| & =\left|\frac{1}{\left|L\left(y^{\prime}\right)\right|} \sum_{x^{\prime} \in L\left(y^{\prime}\right)} f\left(x^{\prime}\right)-\frac{1}{|L(y)|} \sum_{x \in L(y)} f(x)\right| \\
& =\left|\frac{1}{\left|L\left(y^{\prime}\right)\right|} \sum_{x \in L(y)} \sum_{x^{\prime} \in r^{-1}(x)} f\left(x^{\prime}\right)-\frac{1}{|L(y)|} \sum_{x \in L(y)} f(x)\right| \\
& =\left|\frac{1}{n|L(y)|} \sum_{x \in L(y)} \sum_{x^{\prime} \in r^{-1}(x)} f\left(x^{\prime}\right)-\frac{1}{|L(y)|} \sum_{x \in L(y)} f(x)\right| \\
& =\left|\frac{1}{|L(y)|} \sum_{x \in L(y)}\left(\left(\frac{1}{n} \sum_{x^{\prime} \in r^{-1}(x)} f\left(x^{\prime}\right)\right)-f(x)\right)\right| \\
& =\left|\frac{1}{|L(y)|} \sum_{x \in L(y)}\left(\frac{1}{n} \sum_{x^{\prime} \in r^{-1}(x)}\left(f\left(x^{\prime}\right)-f(x)\right)\right)\right| \\
& \leq \frac{1}{|L(y)|} \sum_{x \in L(y)}\left(\frac{1}{n} \sum_{x^{\prime} \in r^{-1}(x)}\left|f\left(x^{\prime}\right)-f(x)\right|\right) \\
& <\frac{1}{|L(y)|} \sum_{x \in L(y)}\left(\frac{1}{n} \sum_{x^{\prime} \in r^{-1}(x)} \varepsilon\right)=\varepsilon .
\end{aligned}
$$

Lemma 6. (a) Let $g: L \rightarrow K$ be a continuous surjection between compact spaces which admits a regular averaging operator and let $M$ be a closed subset of $K$. Then the restriction $g: g^{-1}(M) \rightarrow M$ also admits a regular averaging operator [1, Proposition 18].

(b) Let $\left\{g_{i}: L_{i} \rightarrow K_{i}\right\}$ be a family of continuous surjections between compact spaces which admit regular averaging operators. Then the product map $\prod g_{i}: \prod L_{i} \rightarrow \prod K_{i}$ also admits a regular averaging operator [9, Proposition 4.7]. 
Proof of Theorem 4. Observe that the space $B(\Gamma)$ can be embedded into $B^{+}(\Gamma \times\{a, b\}) \sim B^{+}(\Gamma)$ by the map $u(x)_{\gamma, a}=\max \left(0, x_{\gamma}\right)$ and $u(x)_{\gamma, b}=$ $\max \left(0,-x_{\gamma}\right)$. This allows us to consider $K$ as a subset of $B^{+}(\Gamma)$ with $|\Gamma|=\kappa$. Let $\phi:\{0,1\}^{\omega} \rightarrow[0,1]$ be given by $\phi(x)=\sum r_{i} x_{i}$ where $r_{i}=\frac{1}{3}\left(\frac{2}{3}\right)^{i}$. It is proven in [1] that $\phi$ admits a regular averaging operator and hence by Lemma 6 also $\phi^{\Gamma}:\{0,1\}^{\omega \times \Gamma} \rightarrow[0,1]^{\Gamma}$ and its restriction $\phi^{\Gamma}: L^{\prime}=$ $\left(\phi^{\Gamma}\right)^{-1}(K) \rightarrow K$ admit a regular averaging operator. The space $L^{\prime}$ is a subspace of $L_{0}=\left(\phi^{\Gamma}\right)^{-1}\left(B^{+}(\Gamma)\right)$ with the following description:

$$
\begin{aligned}
x \in L_{0} & \Leftrightarrow \phi^{\Gamma}(x) \in B^{+}(\Gamma) \Leftrightarrow \sum_{\gamma \in \Gamma} \phi^{\Gamma}(x)_{\gamma} \leq 1 \\
& \Leftrightarrow \sum_{\gamma \in \Gamma} \sum_{n=0}^{\infty} r_{n} x_{(\gamma, n)} \leq 1 \Leftrightarrow \sum_{n=0}^{\infty} r_{n} N_{n}(x) \leq 1,
\end{aligned}
$$

where $N_{n}(x)$ is the cardinality of $\operatorname{supp}\left(\left.x\right|_{\Gamma \times\{n\}}\right)$. From this description, if $M_{n}$ denotes the integer part of $r_{n}^{-1}$, then $L^{\prime} \subset L_{0} \subset \prod_{n=1}^{\infty} \sigma_{M_{n}}(\Gamma)$. Theorem 5 and Lemma 6(b) yield the existence of a continuous surjection $g: \sigma_{1}(\Gamma)^{\omega} \rightarrow$ $\prod_{n=1}^{\infty} \sigma_{M_{n}}(\Gamma)$ which admits a regular averaging operator. Making use of Lemma 6(a) we get a surjection $g: L=g^{-1}\left(L^{\prime}\right) \rightarrow L^{\prime}$ with a regular averaging operator, and the composition $L \rightarrow L^{\prime} \rightarrow K$ is the desired map.

We shall now need the so called Pełczyński's decomposition method, which is used to establish the existence of isomorphisms between Banach spaces. For Banach spaces $X$ and $Y$ we shall write $X \mid Y$ if there exists a Banach space $Z$ such that $X \oplus Z$ is isomorphic to $Y$, briefly $X \oplus Z \sim Y$. Also, $Y=\left(X_{1} \oplus X_{2} \oplus \cdots\right)_{c_{0}}$ denotes the $c_{0}$-sum of the Banach spaces $X_{1}, X_{2}, \ldots$,

$$
Y=\left\{y=\left(x_{n}\right) \in \prod X_{n}: \lim \left\|x_{n}\right\|=0\right\}, \quad\|y\|=\sup _{n}\left\|x_{n}\right\| .
$$

Theorem 7 (cf. $[9, \S 8])$. Let $X$ and $Y$ be Banach spaces such that $X \mid Y$, $Y \mid X$ and $(X \oplus X \oplus \cdots)_{c_{0}} \sim X$. Then $X \sim Y$.

If there exists a surjection $\phi: L \rightarrow K$ with a regular averaging operator, then $C(K) \mid C(L)$ (cf. [9]). In particular, if $L \subset K$ is a retract of $K$, then the restriction operator is a regular averaging operator for the retraction. On the other hand, in order to guarantee the last hypothesis in Theorem 7 we shall use the criterion of Lemma 8 below. For topological spaces $K_{n}$, $K_{1} \oplus K_{2} \oplus \cdots$ denotes the discrete topological sum, while $\alpha(S)$ is the onepoint compactification of a locally compact space $S$.

Lemma 8. Let $K$ be a compact space homeomorphic to $\alpha(K \oplus K \oplus \cdots)$. Then $(C(K) \oplus C(K) \oplus \cdots)_{c_{0}} \sim C(K)$. 
Proof. We apply Theorem 7 to $X=(C(K) \oplus C(K) \oplus \cdots)_{c_{0}}$ and $Y=$ $C(K)$. The only point is to check that $X \mid Y$. Let $\infty$ denote the infinity point of $\alpha(K \oplus K \oplus \cdots) \sim K$. Then $X \sim Y^{\prime}=\{f \in C(K): f(\infty)=0\}$ and $Y \sim Y^{\prime} \oplus \mathbb{R}$.

Proof of Theorem 2. Set $K=\sigma_{1}(\Gamma)^{\omega}$ and $L=\prod \sigma_{k_{n}}(\Gamma)$. We apply Theorem 7 to $X=C(K)$ and $Y=C(L)$. First, we have already observed that Theorem 5 and Lemma 6(b) imply the existence of a surjection $f: K$ $\rightarrow L$ with a regular averaging operator, and hence $C(L) \mid C(K)$. On the other hand, $K$ is a retract of $L$ because for any $k, \sigma_{1}(\Gamma)$ is homeomorphic to a clopen subset of $\sigma_{k}(\Gamma)$, the family of all subsets which contain fixed elements $\gamma_{1}, \ldots, \gamma_{k-1}$. Therefore $C(K) \mid C(L)$. By Lemma 8 , it only remains to show that $\alpha(K \oplus K \oplus \cdots) \sim K$. For this, fix $\gamma \in \Gamma$ and set, for $n=1,2, \ldots$,

$$
K_{n}=\left\{x \in K=\sigma_{1}(\Gamma)^{\omega}: \gamma \in x_{1} \cap \cdots \cap x_{n-1} \backslash x_{n}\right\} .
$$

The sets $K_{n}$ are disjoint clopen sets homeomorphic to $K$, and $K$ is the onepoint compactification of their union with infinity point $(\{\gamma\},\{\gamma\}, \ldots)$.

2. Topological classification. This section is devoted to the proof of Theorem 1. Before entering it, we point out why we assume $\Gamma$ to be uncountable. In the countable case, the reasonings below do not apply and the situation is indeed completely different. All perfect totally disconnected metrizable compact spaces are homeomorphic [5, Theorem 7.4] and this implies that all countable products of spaces $\sigma_{k}(\omega)$ are homeomorphic. The finite products are countable compacta, whose topological classification is also well known after the classical paper [7]: two of them are homeomorphic if and only if they have the same Cantor-Bendixson derivation index and the same cardinality of the last nonempty Cantor-Bendixson derivative. Straightforward computations show that these two invariants for a finite product $\prod_{i=1}^{n} \sigma_{k_{i}}(\omega)$ take the values $1+\sum_{i=1}^{n} k_{i}$ and 1 respectively. From now on, $\Gamma$ will always be an uncountable set.

Lemma 9. If $m<n$ then $\sigma_{m}(\Gamma) \times \sigma_{n}(\Gamma)^{\omega}$ is homeomorphic to $\sigma_{n}(\Gamma)^{\omega}$.

Proof. We denote again by $\left(X_{1} \oplus X_{2} \oplus \cdots\right)$ the discrete topological sum of the spaces $X_{1}, X_{2}, \ldots$ and by $\alpha X$ the one-point compactification of the locally compact space $X$. Fix $\gamma_{0}, \ldots, \gamma_{n-1} \in \Gamma$. We consider the set $L=$ $\omega \times\{0, \ldots, n-1\}$ endowed with the lexicographical order: $(k, i)<\left(k^{\prime}, i^{\prime}\right)$ whenever either $k<k^{\prime}$, or $k=k^{\prime}$ and $i<i^{\prime}$. For every $(k, i) \in L$ we define a clopen subset of $\sigma_{n}(\Gamma)^{\omega}$ as

$$
\begin{aligned}
A_{(k, i)} & =\left\{x \in \sigma_{n}(\Gamma)^{\omega}: \gamma_{i} \notin x_{k}, \gamma_{i^{\prime}} \in x_{k^{\prime}} \forall\left(k^{\prime}, i^{\prime}\right)<(k, i)\right\} \\
& =\left\{x \in \sigma_{n}(\Gamma)^{\omega}: \gamma_{i} \notin x_{k} \supset\left\{\gamma_{0}, \ldots, \gamma_{i-1}\right\}, x_{j}=\left\{\gamma_{0}, \ldots, \gamma_{n-1}\right\} \forall j<k\right\} .
\end{aligned}
$$


Notice that $A_{(k, i)}$ is homeomorphic to $\sigma_{n-i}(\Gamma) \times \sigma_{n}(\Gamma)^{\omega}$ and that $\left\{A_{l}: l \in L\right\}$ constitutes a disjoint sequence of clopen subsets of $\sigma_{n}(\Gamma)^{\omega}$ with the only limit point being the sequence $\xi \in \sigma_{n}(\Gamma)^{\omega}$ constantly equal to $\left\{\gamma_{0}, \ldots, \gamma_{n-1}\right\}$. Hence,

$$
\sigma_{n}(\Gamma)^{\omega} \approx \alpha\left(\bigoplus_{l \in L} A_{l}\right) \approx \alpha\left(\bigoplus_{i=0}^{n-1} \bigoplus_{j<\omega}\left(\sigma_{n-i}(\Gamma) \times \sigma_{n}(\Gamma)^{\omega}\right)\right) .
$$

On the other hand, we can perform a similar decomposition in $\sigma_{m}(\Gamma) \times$ $\sigma_{n}(\Gamma)^{\omega}$, defining, for $j<m$ and $(k, i) \in L$,

$$
\begin{aligned}
B_{j}^{\prime}=\left\{(y, x) \in \sigma_{m}(\Gamma) \times \sigma_{n}(\Gamma)^{\omega}: \gamma_{j} \notin y,\left\{\gamma_{0}, \ldots, \gamma_{j-1}\right\} \subset y\right\}, \\
B_{(k, i)}=\left\{(y, x) \in \sigma_{m}(\Gamma) \times \sigma_{n}(\Gamma)^{\omega}: \gamma_{i} \notin x_{k}, \gamma_{i^{\prime}} \in x_{k^{\prime}} \forall\left(k^{\prime}, i^{\prime}\right)<(k, i),\right. \\
\left.\left\{\gamma_{0}, \ldots, \gamma_{m-1}\right\} \subset y\right\} .
\end{aligned}
$$

Again $B_{j}^{\prime}$ is homeomorphic to $\sigma_{m-j}(\Gamma) \times \sigma_{n}(\Gamma)^{\omega}, B_{(k, i)}$ is homeomorphic to $\sigma_{n-i}(\Gamma) \times \sigma_{n}(\Gamma)^{\omega}$ and altogether they constitute a disjoint sequence of clopen sets with a unique limit point $\left(\left\{\gamma_{0}, \ldots, \gamma_{m-1}\right\}, \xi\right)$ not belonging to them, so

$\sigma_{m}(\Gamma) \times \sigma_{n}(\Gamma)^{\omega} \approx \alpha\left(\bigoplus_{l \in L} B_{l} \oplus \bigoplus_{j=0}^{m-1} B_{j}^{\prime}\right) \approx \alpha\left(\bigoplus_{i=0}^{n-1} \bigoplus_{j<\omega}\left(\sigma_{n-i}(\Gamma) \times \sigma_{n}(\Gamma)^{\omega}\right)\right)$.

Lemma 10. If $m<n<\omega$ then $\sigma_{m}(\Gamma)^{\omega} \times \sigma_{n}(\Gamma)^{\omega}$ is homeomorphic to $\sigma_{n}(\Gamma)^{\omega}$.

Proof. We have

$$
\sigma_{m}(\Gamma)^{\omega} \times \sigma_{n}(\Gamma)^{\omega} \approx\left(\sigma_{m}(\Gamma) \times \sigma_{n}(\Gamma)^{\omega}\right)^{\omega} \approx\left(\sigma_{n}(\Gamma)^{\omega}\right)^{\omega} \approx \sigma_{n}(\Gamma)^{\omega} .
$$

LEMMA 11. Let $m_{1}, \ldots, m_{r}<n<\omega$ and $e_{1}, \ldots, e_{r} \leq \omega$. Then the space $\prod_{i=1}^{r} \sigma_{m_{i}}(\Gamma)^{e_{i}} \times \sigma_{n}(\Gamma)^{\omega}$ is homeomorphic to $\sigma_{n}(\Gamma)^{\omega}$.

Proof. Follows from repeated application of Lemmas 9 and 10 above.

From Lemma 11 it follows that any space $\sigma_{\tau}(\Gamma)$ with $i(\tau)=\omega$ is homeomorphic to $\sigma_{(\omega, \omega, \ldots)}(\Gamma)$ (because we can substitute each factor $\sigma_{n}(\Gamma)^{\omega}$ of $\sigma_{\tau}(\Gamma)$ by the homeomorphic $\left.\prod_{i \leq n} \sigma_{i}(\Gamma)^{\omega}\right)$, and this proves part (2) of Theorem 1. Lemma 11 also shows that the values $\tau_{n}$ for $n<i(\tau)$ are irrelevant to the homeomorphism class of $\sigma_{\tau}(\Gamma)$. Hence, in order to prove part (1) of Theorem 1 it remains to show that if $j(\tau)<\omega$ and $\sigma_{\tau}(\Gamma)$ is homeomorphic to $\sigma_{\tau^{\prime}}(\Gamma)$ then $\tau_{n}=\tau_{n}^{\prime}$ for all $n>i(\tau)$.

We recall that a family $\left\{S_{\eta}\right\}_{\eta \in H}$ of sets is a $\Delta$-system if there is a set $S$ (called the root of the $\Delta$-system) such that $S_{\eta} \cap S_{\eta^{\prime}}=S$ for all $\eta \neq \eta^{\prime}$. We will make use of the fact that any uncountable family of finite sets has an uncountable subfamily which is a $\Delta$-system (cf. [4, Theorem 1.4] for $\kappa=\omega$ and $\left.\alpha=\omega_{1}\right)$. 
The following lemma includes as a particular case the fact that $\sigma_{n+1}(\Lambda)$ does not embed into $\sigma_{n}(\Gamma)^{\omega}$. This fact, whose proof corresponds to Steps 1-3 below, was shown to us by Witold Marciszewski, and it seems that it was known to several people before.

Lemma 12. If $|\Lambda|>\omega, n \geq 0, k \geq 0$, then the space $\sigma_{n+1}(\Lambda)^{k+1}$ does not embed into $\sigma_{n}(\Gamma)^{\omega} \times \sigma_{n+1}(\Gamma)^{k}$.

Proof. Suppose that there exists such an embedding.

STEP 1. Passing to a suitable uncountable subset of $\Lambda$, we can suppose that there is an embedding

$$
\phi: \sigma_{n+1}(\Lambda)^{k+1} \rightarrow \sigma_{n}(\Gamma)^{m} \times \sigma_{n+1}(\Gamma)^{k}
$$

for some $m<\omega$. To see this, let $\varphi: \sigma_{n+1}(\Lambda)^{k+1} \rightarrow \sigma_{n}(\Gamma)^{\omega} \times \sigma_{n+1}(\Gamma)^{k}$ be our original embedding. In this step, we shall denote an element $x \in \sigma_{n+1}(\Lambda)^{k+1}$ as $x=\left(x_{0}, \ldots, x_{k}\right)$. For each $\lambda \in \Lambda$ and every $i \in\{0, \ldots, k\}$ we find a clopen subset $A_{\lambda}^{i}$ of $\sigma_{n}(\Gamma)^{\omega} \times \sigma_{n+1}(\Gamma)^{k}$ which separates the disjoint compact sets $\varphi\left(\left\{x: \lambda \in x_{i}\right\}\right)$ and $\varphi\left(\left\{x: \lambda \notin x_{i}\right\}\right)$. Associated to $A_{\lambda}^{i}$ we have a finite

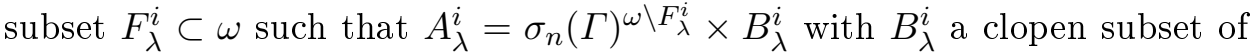

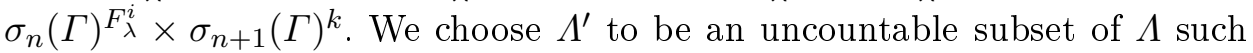
that $\bigcup_{i=0}^{k} F_{\lambda}^{i}=\bigcup_{i=0}^{k} F_{\lambda^{\prime}}^{i}=F$ for all $\lambda, \lambda^{\prime} \in \Lambda^{\prime}$; in this case the composition

$$
\sigma_{n+1}\left(\Lambda^{\prime}\right)^{k+1} \hookrightarrow \sigma_{n+1}(\Lambda)^{k+1} \rightarrow \sigma_{n}(\Gamma)^{\omega} \times \sigma_{n+1}(\Gamma)^{k} \rightarrow \sigma_{n}(\Gamma)^{F} \times \sigma_{n+1}(\Gamma)^{k}
$$

is one-to-one. The reason is that if $x, y \in \sigma_{n+1}\left(\Lambda^{\prime}\right)^{k+1}$ are different then there exist $i \in\{0, \ldots, k\}$ and $\lambda \in \Lambda^{\prime}$ such that $\lambda \in x_{i}$ but $\lambda \notin y_{i}$ (or vice versa). Then $\phi(x) \in A_{\lambda}^{i}$ and $\phi(y) \notin A_{\lambda}^{i}$ so either the coordinate of $\sigma_{n+1}(\Gamma)^{k}$ or some coordinate of $F_{i}^{\lambda} \subset F$ must be different for $\phi(x)$ and $\phi(y)$.

STEP 2. For $i=0, \ldots, k$ and $\lambda \in \Lambda$ we define $e_{i}^{\lambda} \in \sigma_{n+1}(\Lambda)^{k+1}$ to be the element which has $\{\lambda\}$ in coordinate $i$ and $\emptyset$ in all other coordinates. Each $\phi\left(e_{i}^{\lambda}\right)$ will be of the form

$$
\phi\left(e_{i}^{\lambda}\right)=\left(x_{i}^{\lambda}[1], \ldots, x_{i}^{\lambda}[m], x_{i}^{\lambda}[m+1], \ldots, x_{i}^{\lambda}[m+k]\right)
$$

with $x_{i}^{\lambda}[j] \in \sigma_{n}(\Gamma)$ if $j \leq m$ and $x_{i}^{\lambda}[j] \in \sigma_{n+1}(\Gamma)$ if $m<j \leq m+k$. Passing to a suitable uncountable subset of $\Lambda$, we can assume that for every fixed $i \in\{0, \ldots, k\}$ and $j \in\{1, \ldots, m+k\}$ the family $\left\{x_{i}^{\lambda}[j]: \lambda \in \Lambda\right\}$ is a $\Delta$-system of root $R_{i}[j]$ formed by sets of the same cardinality $c_{i}[j]$.

STEP 3. We claim that for $i=0, \ldots, n$ and $j=1, \ldots, m$, the $\Delta$-system $\left\{x_{i}^{\lambda}[j]: \lambda \in \Lambda\right\}$ is constant. Suppose the contrary for some fixed $i \leq n$ and $j \leq m$. Then $x_{i}^{\lambda}[j]=R \cup S^{\lambda} \in \sigma_{n}(\Gamma)$ where $R \cap S^{\lambda}=\emptyset, S^{\lambda} \neq \emptyset$, and $S^{\lambda} \cap S^{\lambda^{\prime}}=\emptyset$ for $\lambda \neq \lambda^{\prime}$. We consider the sets

$$
A_{\lambda}=\left\{y=(y[1], \ldots, y[m+k]) \in \sigma_{n}(\Gamma)^{m} \times \sigma_{n+1}(\Gamma)^{k}: y[j] \supset S^{\lambda}\right\} .
$$


The $A_{\lambda}$ 's are neighborhoods of the $\phi\left(e_{i}^{\lambda}\right)$ 's with the property that for every $F \subset \Lambda$ with $|F|>n, \bigcap_{\lambda \in F} A_{\lambda}=\emptyset$ (because for $y$ in that intersection, $|y[j]|>n$ and $\left.y[j] \in \sigma_{n}(\Gamma)\right)$. Let $\psi: \sigma_{n+1}(\Lambda) \rightarrow \sigma_{n+1}(\Lambda)^{k+1}$ be the map defined by $\psi(x)_{i}=x$ and $\psi(x)_{i^{\prime}}(x)=\emptyset$ if $i^{\prime} \neq i$. Then the $(\phi \psi)^{-1}\left(A_{\lambda}\right)^{\prime}$ s are neighborhoods of the $\{\lambda\}$ 's in $\sigma_{n+1}(\Lambda)$ with $\bigcap_{\lambda \in F}(\phi \psi)^{-1}\left(A_{\lambda}\right)=\emptyset$ for every $F \subset \Lambda$ with $|F|>n$. This is a contradiction since such a family of neighborhoods cannot be found. Namely, take basic neighborhoods with $\{\lambda\} \in \Phi_{\{\lambda\}}^{G_{\lambda}} \subset(\phi \psi)^{-1}\left(A_{\lambda}\right)$ and take $\Lambda^{\prime} \subset \Lambda$ uncountable with $\left\{G_{\lambda}: \lambda \in \Lambda^{\prime}\right\}$ a $\Delta$-system of root $R^{\prime}$. Then construct inductively a finite sequence $F=$ $\left\{\lambda_{1}, \ldots, \lambda_{n+1}\right\} \subset \Lambda^{\prime} \backslash R^{\prime}$ such that $\lambda_{p} \notin \bigcup_{q<p} G_{\lambda_{q}}$ and $G_{\lambda_{p}} \cap\left\{\lambda_{1}, \ldots, \lambda_{p-1}\right\}=$ $\emptyset$ (notice that it is possible to choose such a $\lambda_{p}$ because $\left\{\lambda_{1}, \ldots, \lambda_{p-1}\right\} \cap$ $R^{\prime}=\emptyset$ and hence there are only finitely many $G_{\lambda}$ 's with $\lambda \in \Lambda^{\prime}$ and $G_{\lambda} \cap$ $\left.\left\{\lambda_{1}, \ldots, \lambda_{p-1}\right\} \neq \emptyset\right)$. In this case we have $F \in \bigcap_{\lambda \in F}(\phi \psi)^{-1}\left(A_{\lambda}\right)$.

STEP 4. Notice that for $k=0$ we have already arrived at a contradiction and the proof is complete. When $k>0$ we need some extra work. From Step 3, we deduce that for each $i \in\{0, \ldots, k\}$ there must exist $j \in\{m+1$, $\ldots, m+k\}$ such that the family $\left\{x_{i}^{\lambda}[j]: \lambda \in \Lambda\right\}$ is a nonconstant $\Delta$-system. Since $i$ runs through a set of $k+1$ elements and $j$ through a set of $k$ elements, there must exist two different $i, i^{\prime} \in\{0, \ldots, k\}$ such that for the same $j$, $\left\{x_{i}^{\lambda}[j]: \lambda \in \Lambda\right\}$ and $\left\{x_{i^{\prime}}^{\lambda}[j]: \lambda \in \Lambda\right\}$ are nonconstant $\Delta$-systems. We assume that $c_{i}[j] \geq c_{i^{\prime}}[j]$ (these numbers are defined in Step 2). Again, for $\lambda \in \Lambda$ we consider the sets

$$
\begin{aligned}
& A_{\lambda}=\left\{(y[1], \ldots, y[m+k]) \in \sigma_{n}(\Gamma)^{m} \times \sigma_{n+1}(\Gamma)^{k}: y[j] \supset x_{i}^{\lambda}[j]\right\}, \\
& A_{\lambda}^{\prime}=\left\{(y[1], \ldots, y[m+k]) \in \sigma_{n}(\Gamma)^{m} \times \sigma_{n+1}(\Gamma)^{k}: y[j] \supset x_{i^{\prime}}^{\lambda}[j]\right\} .
\end{aligned}
$$

The $A_{\lambda}$ 's and the $A_{\lambda}^{\prime}$ 's are neighborhoods of the $\phi\left(e_{i}^{\lambda}\right)$ 's and the $\phi\left(e_{i^{\prime}}^{\lambda}\right)$ 's respectively with the property that

$$
\forall \lambda \in \Lambda \forall F \subset \Lambda \quad\left(|F|>n \wedge x_{i}^{\lambda}[j] \nsubseteq \bigcup_{\mu \in F} x_{i^{\prime}}^{\mu}[j]\right) \Rightarrow A_{\lambda} \cap \bigcap_{\mu \in F} A_{\mu}^{\prime}=\emptyset .
$$

That intersection is empty because if $y$ belongs to it, then

$$
x_{i}^{\lambda}[j] \cup \bigcup_{\mu \in F} x_{i^{\prime}}^{\mu}[j] \subset y[j] \in \sigma_{n+1}(\Gamma)
$$

and the set on the left, if $x_{i}^{\lambda}[j] \nsubseteq \bigcup_{\mu \in F} x_{i^{\prime}}^{\mu}[j]$, has cardinality greater than $n+1$, a contradiction. Since the $\Delta$-systems are not constant and $c_{i}[j] \geq c_{i^{\prime}}[j]$, it follows that if $x_{i}^{\lambda}[j] \subseteq \bigcup_{\mu \in F} x_{i^{\prime}}^{\mu}[j]$ holds, there must be some $\mu \in F$ and $\gamma \in x_{i}^{\lambda}[j]$ such that $\gamma \in x_{i^{\prime}}^{\mu}[j] \backslash R_{i^{\prime}}[j]$. For a fixed $\lambda$ there are only finitely many $\mu$ 's with $\left(x_{i^{\prime}}^{\mu}[j] \backslash R_{i^{\prime}}[j]\right) \cap x_{i}^{\lambda}[j] \neq \emptyset$. Hence for every $\lambda$, we can find a cofinite subset $\Lambda_{\lambda}$ of $\Lambda$ such that the hypothesis $x_{i}^{\lambda}[j] \nsubseteq \bigcup_{\mu \in F} x_{i^{\prime}}^{\mu}[j]$ of statement $(*)$ holds whenever $F \subset \Lambda_{\lambda}$. For short, we know that for every 
$\lambda \in \Lambda$ there exists a cofinite subset $\Lambda_{\lambda}$ of $\Lambda$ such that

$$
\forall F \subset \Lambda_{\lambda} \quad|F|>n \Rightarrow A_{\lambda} \cap \bigcap_{\mu \in F} A_{\mu}^{\prime}=\emptyset .
$$

This contradicts the following lemma for $B_{\lambda}=\phi^{-1}\left(A_{\lambda}\right)$ and $B_{\lambda}^{\prime}=\phi^{-1}\left(A_{\lambda}^{\prime}\right)$ :

Lemma 13. For every $\lambda \in \Lambda$, let $B_{\lambda}$ and $B_{\lambda}^{\prime}$ be neighborhoods of $e_{i}^{\lambda}$ and $e_{i^{\prime}}^{\lambda}$ respectively in $\sigma_{n+1}(\Lambda)^{k+1}$. Then there exists $\lambda_{0} \in \Lambda$ and an infinite set $S \subset \Lambda$ such that for every $F \subset S$ with $|F|=n+1$,

$$
B_{\lambda_{0}} \cap \bigcap_{\mu \in F} B_{\mu}^{\prime} \neq \emptyset \text {. }
$$

Proof. For a simpler notation, we will assume that $i=0$ and $i^{\prime}=1$. Notice that a basic clopen subset $\Phi_{F}^{G}$ of $\sigma_{n+1}(\Lambda)$ is nonempty if and only if $F \cap G=\emptyset$ and $|F| \leq n+1$. Each $B_{\lambda}$ and each $B_{\mu}^{\prime}$ contain basic clopen sets of the form

$$
\begin{gathered}
\Phi_{\{\lambda\}}^{G_{0}^{\lambda}} \times \Phi_{\emptyset}^{G_{1}^{\lambda}} \times \Phi_{\emptyset}^{G_{2}^{\lambda}} \times \cdots \times \Phi_{\emptyset}^{G_{k}^{\lambda}} \subseteq B_{\lambda}, \\
\Phi_{\emptyset}^{H_{0}^{\mu}} \times \Phi_{\{\mu\}}^{H_{1}^{\mu}} \times \Phi_{\emptyset}^{H_{2}^{\mu}} \times \cdots \times \Phi_{\emptyset}^{H_{k}^{\mu}} \subseteq B_{\mu}^{\prime},
\end{gathered}
$$

with all $G_{l}^{\lambda}$ and $H_{l}^{\mu}$ finite subsets of $\Lambda$ and $\lambda \notin G_{0}^{\lambda}$ and $\mu \notin H_{1}^{\mu}$. First, we find a countably infinite set $M \subset \Lambda$ such that $\mu^{\prime} \notin H_{1}^{\mu}$ for all $\mu, \mu^{\prime} \in M$. This can be done as follows. We begin with an infinite $M_{1} \subset \Lambda$ such that the family $\left\{H_{1}^{\mu}: \mu \in M_{1}\right\}$ is a $\Delta$-system of root $R$, and we set $M_{2}=M_{1} \backslash R$. Then we can find recursively a sequence $\left(\mu_{p}\right)_{p<\omega} \subset M_{2}$ such that $\mu_{p} \notin \bigcup_{q<p} H_{1}^{\mu_{q}}$ and $H_{1}^{\mu_{p}} \cap\left\{\mu_{1}, \ldots, \mu_{p-1}\right\}=\emptyset$. Next, we set $M=\left\{\mu_{p}: p<\omega\right\}$. Now, we choose $\lambda_{0} \notin \bigcup_{\mu \in M} H_{0}^{\mu}$ and set $S=\left\{\mu \in M: \mu \notin G_{1}^{\lambda_{0}}\right\}$. Then $\lambda_{0}$ and $S$ are as desired. Namely, take $F \subset S$ with $|F|=n+1$, and for every $j=0, \ldots, k$ define $I_{j}=G_{j}^{\lambda_{0}} \cup \bigcup_{\mu \in F} H_{j}^{\mu}$ so that

$$
B_{\lambda_{0}} \cap \bigcap_{\mu \in F} B_{\mu}^{\prime} \supset \Phi_{\left\{\lambda_{0}\right\}}^{I_{0}^{\mu}} \times \Phi_{F}^{I_{1}^{\mu}} \times \prod_{j=2}^{k} \Phi_{\emptyset}^{I_{j}^{\mu}} .
$$

On the one hand, $\Phi_{\left\{\lambda_{0}\right\}}^{I_{0}^{\mu}} \neq \emptyset$ because we have chosen $\lambda_{0} \notin \bigcup_{\mu \in M} H_{0}^{\mu}$, so $\lambda_{0} \notin I_{0}^{\mu}$. On the other hand, $\Phi_{F}^{I_{1}^{\mu}} \neq \emptyset$ because, first, since $F \subset M$ and $\mu^{\prime} \notin H_{1}^{\mu}$ for all $\mu, \mu^{\prime} \in M$, it follows that $F \cap \bigcup_{\mu \in F} H_{1}^{\mu}=\emptyset$, and second, since $F \subset S$, just by the definition of $S, F \cap G_{1}^{\lambda_{0}}=\emptyset$.

Lemma 12 implies that $j(\tau)=j\left(\tau^{\prime}\right)$ whenever $\sigma_{\tau}(\Gamma)=\sigma_{\tau^{\prime}}(\Gamma)$, since it shows that $j(\tau)=\omega$ if and only if $\sigma_{n}(\Gamma)$ can be embedded into $\sigma_{\tau}(\Gamma)$ for all $n<\omega$ and, if this is not the case, $j(\tau)$ is the greatest integer $n$ for which $\sigma_{n}(\Gamma)$ embeds into $\sigma_{\tau}(\Gamma)$. Hence, in the situation of part (1) of Theorem 1, it happens that $j(\tau)=j\left(\tau^{\prime}\right)=j$ and moreover that $\tau_{n}=\tau_{n}^{\prime}$ for all $n \geq j$ 
since, by Lemma 12 again, $\tau_{j}=\tau_{j}^{\prime}$ is the greatest integer $k$ such that $\sigma_{j}(\Gamma)^{k}$ embeds into $\sigma_{\tau}(\Gamma)$ and of course, $\tau_{n}=\tau_{n}^{\prime}=0$ for all $n>j$. In order to finish the proof of part (1), we must check that $i(\tau)=i\left(\tau^{\prime}\right)=i$ and that $\tau_{k}=\tau_{k}^{\prime}$ for $i<k<j$. To see this, we shall look at embeddability of spaces $\sigma_{n}(\Gamma)^{k}$ into clopen subsets of $\sigma_{\tau}(\Gamma)$. For this purpose, we observe that it is enough to look at some basic family of clopen sets, if the others are their unions:

LEMMA 14. Let $X$ be a compact space and $C_{1}, \ldots, C_{t}$ open subsets of $X$. If $\sigma_{n}(\Lambda)^{k}$ embeds into $\bigcup_{i=1}^{t} C_{i}$, then there exists $i \leq t$ such that $\sigma_{n}(\Lambda)^{k}$ embeds into $C_{i}$.

Proof. It reduces to proving that whenever we express $\sigma_{n}(\Lambda)^{k}$ as a union of open sets as

$$
\sigma_{n}(\Lambda)^{k}=C_{1} \cup \cdots \cup C_{t}
$$

then some $C_{i}$ must contain a copy of $\sigma_{n}(\Lambda)^{k}$. Pick $i \in\{1, \ldots, t\}$ such that $x_{0}=(\emptyset, \ldots, \emptyset) \in C_{i}$. There are finite subsets $G^{1}, \ldots, G^{k}$ of $\Lambda$ such that

$$
x_{0} \in \Phi_{\emptyset}^{G^{1}} \times \cdots \times \Phi_{\emptyset}^{G^{k}} \subset C_{i} .
$$

This finishes the proof because $\Phi_{\emptyset}^{G^{1}} \times \cdots \times \Phi_{\emptyset}^{G^{k}}$ is homeomorphic to $\sigma_{n}(\Lambda)^{k}$.

Let now $K=\prod_{s \in S} \sigma_{n_{s}}(\Gamma)$ be any finite or countable product of spaces of type $\sigma_{n}(\Gamma)$. Any clopen subset of $K$ is a finite union of basic clopen sets of the form

$$
C=\prod_{s \in A} \Phi_{F_{s}}^{G_{s}} \times \prod_{s \notin A} \sigma_{n_{s}}(\Gamma)
$$

where $A$ is a finite subset of $S$ and $\Phi_{F_{s}}^{G_{s}}$ a basic clopen set of $\sigma_{n_{s}}(\Gamma)$. Such a basic clopen set is homeomorphic to

$$
C \sim \prod_{s \in A} \sigma_{n_{s}-\left|F_{s}\right|}(\Gamma) \times \prod_{s \notin A} \sigma_{n_{s}}(\Gamma) .
$$

Now, by Lemma 12, Lemma 14 and the topological description $(\star)$ of the basic clopen sets given above, we are in a position to state that, in the situation of part (1) of Theorem 1, the following hold:

(A) $i(\tau)=i\left(\tau^{\prime}\right)=i$ is the greatest integer $n$ such that $\sigma_{n}(\Gamma)$ embeds into any clopen subset of $\sigma_{\tau}(\Gamma)$.

(B) For $n=j, j-1, j-2, \ldots, i+1, \tau_{n}=\tau_{n}^{\prime}$ is the greatest integer $k$ such that there is a clopen subset $C$ of $\sigma_{\tau}(\Gamma)$ in which $\sigma_{n+1}(\Gamma)$ cannot be embedded, but in which nevertheless $\sigma_{n}(\Gamma)^{k+\sum_{r>n} \tau_{r}}$ does embed.

This finishes the proof of Theorem 1. For statement $(\mathrm{A})$, since $\sigma_{i(\tau)}(\Gamma)^{\omega}$ is one of the factors of $\sigma_{\tau}(\Gamma)$, it is clear that $\sigma_{i(\tau)}(\Gamma)^{\omega}$ is still a factor of any clopen set like in $(\star)$. On the other hand, there are only finitely many factors of type $\sigma_{m}(\Gamma), m>i(\tau)$, in $\sigma_{\tau}(\Gamma)$, hence a clopen set like in $(\star)$ can be obtained so that all factors in $\prod_{s \in A} \sigma_{n_{s}-\left|F_{s}\right|}(\Gamma) \times \prod_{s \notin A} \sigma_{n_{s}}(\Gamma)$ are of the 
form $\sigma_{m}(\Gamma)$ with $m \leq i(\tau)$. By Lemma $12, \sigma_{k}(\Gamma)$ does not embed in such $C$ if $k>i(\tau)$.

Statement (B) is proved by "downward induction" starting at $j$ and finishing at $i+1$. We know, by Lemma 11, that

$$
\sigma_{\tau}(\Gamma) \sim \sigma_{i}(\Gamma)^{\omega} \times \prod_{m=i+1}^{j} \sigma_{m}(\Gamma)^{\tau_{m}}
$$

Now statement (B) for $n=j$ is a direct consequence of Lemma 12 since no clopen set can contain $\sigma_{j+1}(\Gamma)$ and the maximal exponent of $\sigma_{j}(\Gamma)$ inside $\sigma_{\tau}(\Gamma)$ is $\tau_{j}$. We pass to the case when $i<n<j$. The "biggest" possible basic clopen subset $C$ of $\sigma_{\tau}(\Gamma)$ not containing $\sigma_{n+1}(\Gamma)$ is obtained by reducing if necessary the factors $\sigma_{m}(\Gamma)$ with $m>n$ :

$$
C \sim \sigma_{i}(\Gamma)^{\omega} \times \prod_{m=i+1}^{n} \sigma_{m}(\Gamma)^{\tau_{m}} \times \prod_{m=n+1}^{j} \sigma_{n}(\Gamma)^{\tau_{m}}
$$

The maximal exponent of $\sigma_{n}(\Gamma)$ in such a $C$ is $\sum_{m=n}^{j} \sigma_{\tau_{m}}$.

The present work was written during a visit to the University of Warsaw. The author wishes to thank for the hospitality, specially to Witold Marciszewski and Roman Pol, and to Rafał Górak from the Polish Academy of Sciences. This work owes very much to the discussion with them and their suggestions.

\section{References}

[1] S. A. Argyros and A. D. Arvanitakis, A characterization of regular averaging operators and its consequences, Studia Math. 151 (2002), 207-226.

[2] Y. Benyamini, M. E. Rudin, and M. Wage, Continuous images of weakly compact subsets of Banach spaces, Pacific J. Math. 70 (1977), 309-324.

[3] Y. Benyamini and T. Starbird, Embedding weakly compact sets into Hilbert space, Israel J. Math. 23 (1976), 137-141.

[4] W. W. Comfort and S. A. Negrepontis, Chain Conditions in Topology, Cambridge Tracts in Math. 79, Cambridge Univ. Press, Cambridge, 1982.

[5] A. S. Kechris, Classical Descriptive Set Theory, Grad. Texts Math. 156, Springer, New York, 1995.

[6] W. Marciszewski, On Banach spaces $C(K)$ isomorphic to $c_{0}(\Gamma)$, Studia Math. 156 (2003), 295-302.

[7] S. Mazurkiewicz et W. Sierpiński, Contribution à la topologie des ensembles dénombrables, Fund. Math. 1 (1920), 17-27.

[8] A. A. Milyutin, Isomorphism of the spaces of continuous functions over compact sets of the cardinality of the continuum, Teor. Funktsii Funktsional. Anal. i Prilozhen. Vyp. 2 (1966), 150-156 (in Russian).

[9] A. Pełczyński, Linear extensions, linear averagings, and their applications to linear topological classification of spaces of continuous functions, Dissertationes Math. (Rozprawy Mat.) 58 (1968). 
[10] E. V. Shchepin, Topology of limit spaces of uncountable inverse spectra, Uspekhi Mat. Nauk 31 (1976), no. 5, 191-226 (in Russian): English transl.: Russian Math. Surveys 31 (1976), no. 5, 155-191.

Departamento de Matemáticas

Universidad de Murcia

30100 Espinardo (Murcia), Spain

E-mail: avileslo@um.es

Received 14 February 2005;

in revised form 30 June 2005 